REVUE DE L'INSTITUT

FRANÇAIS D'HISTOIRE

EN ALLEMAGNE

\section{Revue de l'IFHA}

Revue de l'Institut français d'histoire en Allemagne

$2 \mid 2010$

IFHA 2

\title{
Les commémorations historiques en 2009
}

\section{Marion Deschamp et Thomas Lienhard}

\section{OpenEdition}

\section{Journals}

Édition électronique

URL : http://journals.openedition.org/ifha/242

DOI : 10.4000/ifha.242

ISSN : 2198-8943

\section{Éditeur}

IFRA - Institut franco-allemand (sciences historiques et sociales)

\section{Édition imprimée}

Date de publication : 1 janvier 2010

Pagination : 121-132

ISSN : 2190-0078

\section{Référence électronique}

Marion Deschamp et Thomas Lienhard, «Les commémorations historiques en 2009 », Revue de l'IFHA [En ligne], 2 | 2010, mis en ligne le 01 février 2013, consulté le 03 mai 2019. URL : http:// journals.openedition.org/ifha/242 ; DOI : 10.4000/ifha.242

Ce document a été généré automatiquement le 3 mai 2019.

(CIFHA 


\title{
Les commémorations historiques en 2009
}

\author{
Marion Deschamp et Thomas Lienhard
}

1 À la frénésie commémorative qui s'est emparée de nos sociétés se sont associées les histoires mémorielles: la multiplication de colloques, journées d'études, tables rondes, cycles de conférences et surtout de publications organisées et publiées à "l'occasion de » (qu'il s'agisse de fêter les 60 ans de carrière d'un vénérable professeur, l'anniversaire d'une société savante ou le centenaire de tel ou tel événement historique) montre bien que le rythme scientifique de la discipline historique se plie aussi souvent, et sans se rebiffer, à l'ordre jubilaire. L'année 2009 en tout cas, qualifiée en Allemagne de " Superjubiläumsjahr ", a apporté un écot non négligeable à la moisson des commémorations et autres évènement du souvenir, nationaux comme internationaux, populaires comme plus académiques. Le complexe jubilaire était particulièrement dense outre-Rhin, avec la célébration de la bataille de Varus qui se déroula il y a deux mille ans dans la forêt de Teutoburg, le jubilé Calvin, le lancement de l'année Darwin (pour le 200ème anniversaire de la naissance de ce dernier et le 150ème de la parution de son maitre-livre, L'Origine des espèces), les 60 ans de la création de la RFA, ou encore les 20 ans de la Chute du Mur. À titre d'exemple, nous présenterons ici comment furent orchestrées deux de ces ensembles mémorio-historiographiques.

\section{La commémoration de la victoire d'Arminius contre Varus (Thomas Liehard)}

2 En l'an 09 de notre ère, un chef de guerre germanique nommé Arminius profita des relations de confiance qu'il avait tissées avec le général romain Varus pour trahir celui-ci et, dans le Teutoburger Wald proche d'Osnabrück, anéantir les trois légions dirigées par l'officier. Deux mille ans plus tard, l'année 2009 fut l'occasion de nombreuses manifestations ou publications allemandes portant sur cet événement. 
3 Il est vrai que l'Allemagne n'en était pas à son coup d'essai en ce domaine, et que la commémoration d'Arminius, germanisé en « Hermann » depuis l'époque de Luther, avait déjà une longue histoire derrière elle ${ }^{1}$. Rappelons-en brièvement les principaux avatars : en 1515, la publication des Annales de Tacite, qui mettaient Arminius en lumière, donna l'occasion aux humanistes allemands d'identifier un héros fondateur antique pour leur espace politique, contrecarrant ainsi l'opinion des nations voisines selon lesquelles l'Allemagne n'avait pas d'Antiquité. Pour le Saint-Empire, Arminius devint ainsi à la fois un premier héros et un libérateur des vertus et libertés germaniques face à Rome. Par la suite, le modèle fourni par ce personnage fut invoqué successivement contre le catholicisme romain puis contre les efforts expansionnistes de la France louisquatorzienne ou napoléonienne. En 1875, l'érection d'une gigantesque statue dédiée au chef de guerre sur le lieu supposé de la bataille contribua à la mémoire de l'événement, tout en ornant Arminius d'une nouvelle facette puisque celui-ci était désormais associé à l'idée d'unité allemande. Après ce clinamen, toutefois, le mythe d'Arminius perdit de son efficacité au XXe siècle et fut moins souvent évoqué, en particulier après 1945.

Qu'en fut-il lors du jubilé de 2009? Tentons d'abord, pour aborder la diffusion de ce thème dans la société allemande actuelle, de présenter les manifestations extrauniversitaires auxquelles donnèrent lieu la bataille du Teutoburger Wald et celui qui l'avait remportée. Parmi les principales chaînes de radio ou de télévision nationales, aucune ne fit l'impasse sur ce sujet, consacrant généralement un minimum de trois émissions à la question au cours de l'année 2009. Auprès des particuliers et en particulier sur Internet, l'anniversaire charria avec lui son lot de représentations passionnées, parfois fort savantes, parfois totalement fantaisistes sur l'Antiquité germanique: on signalera notamment, pour le lecteur amateur d'excentricités, un site cherchant à démontrer, coïncidence chronologique à l'appui, qu'Arminius n'était autre que Jésus. Un jeu vidéo créé pour l'occasion, portant le nom d'Arminius (en lettres gothiques, naturellement), renforcera chez ses usagers l'idée que l'Antiquité, en particulier germanique, était le lieu de combats ultra-violents et de femmes lascives. Même les librairies les plus respectables se prêtèrent parfois au jeu, déposant en vitrine une véritable épée grandeur nature et un crâne à côté des ouvrages qui, en grand nombre également, étaient parus à la même date. En effet, et même si l'on en reste d'abord au domaine extra-universitaire, Arminius engendra en 2009 une petite dizaine de romans dont les auteurs, pour inspirés qu'ils fussent, eurent le flair de publier leur œuvre l'année du bimillénaire. En revanche, il faut noter que les autorités publiques restèrent très largement à distance de ces manifestations : les discours commémoratifs, les tentatives de reconstitution historique, les rassemblements qui avaient accompagné le précédent centenaire en 1909 furent cette fois rarissimes. En 2009, Arminius était un bon thème commercial et suscitait encore bien des commentaires, ce qui lui redonna une certaine actualité dans l'opinion publique; mais il n'était plus affaire de politique ${ }^{2}$.

5 Qu'en fut-il dans le domaine universitaire? Si l'on met à part les rééditions d'ouvrages antérieurs ainsi que les recueils de documents pour écoliers ou pour étudiants, il reste quelque quinze ouvrages de recherche qui furent publiés en 2009 et renouvelèrent ainsi l'historiographie de cet événement. L'ampleur de ces volumes était généralement modeste, mais elle fut compensée par l'organisation simultanée de trois expositions dont l'une, présentée conjointement à Haltern, Detmold et Kalkriese, attira quelque 450000 visiteurs et donna lieu à trois imposants recueils d'articles qui rassemblaient, cette fois, les plus éminents spécialistes de la question et présentaient les thèmes les plus divers ${ }^{3}$. Il 
est vrai que pour l'essentiel, ces volumes, tout comme l'ensemble des publications suscitées en 2009 par Arminius, se caractérisaient par une approche cantonnée à trois champs bien circonscrits : l'un exploitait les résultats récents de l'archéologie dans le Teutoburger Wald, l'autre profitait de cette commémoration pour offrir de nouvelles synthèses à propos de l'empire romain, tandis que le troisième dressait un bilan historiographique des études consacrées à Arminius dans les deux derniers siècles. En revanche, un des thèmes qui avaient fait la fortune du précédent centenaire en 1909, à savoir les liens entre la bataille de l'an 09 et l'identité allemande, fut désormais relégué aux oubliettes de la science. Certes, Angela Merkel se fendit encore d'un avant-propos dans l'un de ces ouvrages, considérant ainsi que pour une part, Arminius relevait encore de la vie politique de l'Allemagne actuelle; mais pour justifier cette intervention, elle se contenta de rappeler, de manière particulièrement évasive, que «l'étude de l'histoire est importante pour nos sociétés ", sans plus évoquer une quelconque forme de filiation entre Arminius et la République Fédérale d'Allemagne. De toute évidence, en 2009, l'heure était à la vigilance dans la commémoration de cet événement: consciente des détournements que les historiens d'autrefois avaient pu faire subir à l'antique chef germanique pour servir des causes discutables, la recherche scientifique comme l'opinion publique considérèrent que la bataille du Teutoburger Wald constituait certes un événement digne de curiosité et d'étude, mais en aucun cas un événement national.

On ne pourra que souscrire à cette prudence scientifique. Toutefois, à regarder de plus près les études parues en 2009 à ce sujet, on acquiert l'impression qu'il y manque quelque chose: il semble qu'en voulant dissocier Arminius et l'Allemagne - réflexe bien compréhensible -, les historiens aient également ostracisé le thème des Germains antiques. Comme on l'a vu plus haut, la plupart des études publiées en 2009 contournaient ce thème, n'évoquant le concept que pour rappeler qu'il fallait s'en méfier. Or malgré la rareté des sources disponibles, il semble qu'il soit encore possible de reconstituer une histoire, certes très lacunaire, de ces sociétés antiques : il existe malgré tout quelques mentions fugaces dans les sources conservées (après tout, rien ne permet d'affirmer que la Germanie de Tacite est exclusivement une compilation de clichés exotiques puisés dans l'ethnographie romaine antérieure), l'archéologie permet de compléter ces données, des sources postérieures permettent parfois de consolider tel ou tel indice, et en confrontant le tableau ainsi obtenu avec les modèles proposés par d'autres disciplines (en particulier l'ethnologie, à propos de la cohérence politique des tribus germaniques), il reste loisible de considérer les sociétés germaniques antiques comme des objets d'histoire. Il aurait ainsi été possible de présenter, même de manière hypothétique et impressionniste, les réseaux sociaux d'Arminius, le sens de l'autorité qui était le sien, ou encore la nature de son identité ethnique. Or ces thèmes furent omis en 2009, de sorte qu'on ne comprend toujours pas, après la lecture de cette vague de publications, pourquoi diable ce chef de guerre prit l'initiative de trahir l'empire, de défier et d'anéantir trois légions romaines.

7 Il est vrai que pour ce silence historiographique, les raisons sont multiples. En premier lieu, la notion de Germains pose des difficultés conceptuelles : il est désormais acquis que les sociétés naguère qualifiées de germaniques n'avaient guère d'unité, et que le terme même, appliqué par César aux peuples situés immédiatement à l'est du Rhin (à moins de $200 \mathrm{~km}$ de celui-ci), est difficilement transposable à tous les groupes qui parlaient une langue définie comme germanique par les linguistes. En second lieu, on a vu plus haut que la notion de Germains avait été employée indûment comme une première étape de l'histoire allemande, ce qui a contribué à la déconsidérer. S'ajoute à cela une polémique 
déjà ancienne qui avait remis en cause le caractère germanique de la révolte de l'an 09 : selon la thèse de Dieter Timpe (rarement retenue, il est vrai), le conflit militaire du Teutoburger Wald n'aurait pas opposé Rome et des peuples extérieurs, mais plusieurs factions au sein même de l'armée romaine, parmi lesquelles des mercenaires dont la germanité était parfois ancienne ${ }^{4}$. On comprend bien que ces divers arguments aient pu inciter à la prudence les auteurs scientifiques qui ont exploré le sujet en 2009. Mais on ne peut s'empêcher de rappeler l'adage juridique médiéval selon lequel abusus usum non tollit, et de considérer qu'il est dommage d'occulter complètement les Germains au nom des détournements dont ces derniers ont fait l'objet. Il aurait ainsi été possible, sans pour autant céder à l'héroïsation, de donner à Arminius un peu d'épaisseur historique et psychologique : cette question, écartelée entre l'approche romaniste, la précision des études archéologiques et les rappels historiographiques, est restée sans réponse.

\section{Calvin-Jubiläum 2009 (Marion Deschamp)}

Inscrit à l'inventaire des commémorations de l'année 2009, le jubilé Calvin, fêtant le 500ème anniversaire de la naissance du réformateur picard dont la vie et l'œuvre sont si fortement associées à l'histoire de l'Église réformée de Genève, a suscité un engouement international. En effet, si le cœur des manifestations, populaires ou érudites, a bien sûr battu dans une Genève transformée un an durant en véritable lieu de mémoire calvinien, la France, mais aussi d'autres pays d'Europe et du monde se sont fortement mobilisés pour l'événement. En Allemagne cependant, le Calvin-Jahr 2009 s'est inscrit dans un contexte particulier, marqué par la concurrence - feutrée - des mémoires confessionnelles et des projets de recherches associées à ces dernières. Le memento calvinien s'est en effet heurté à un Goliath commémoratif, la «Luther-Dekade », lancée en 2007 pour préparer le super-jubilé 2017, fêtant le 500ème anniversaire du début de la Réforme allemande. L'ombre du Reformationsjubiläum plane en tout cas sur une partie de l'histoire religieuse de la période moderne, puisqu'une foule de projets de recherche s'y référant est en cours, soutenue notamment par la Deutsche Forschungsgemeinschaft. L'un de ces projets, intitulé «Geist der Zeiten » et emmené par la faculté de théologie de HalleWittenberg, s'est emparé des jubilés luthériens précédents (de 1617 à 1917) comme objet d'étude per se, dans une sorte d'histoire mémorielle à la puissance deux, elle-même fidèle à «l'esprit du temps » contemporain (où l'histoire semble objectiver non seulement la mémoire mais encore les histoires mémorielles, dans une sorte de redoublement réflexif). En ce sens, 2009, année Calvin, a pu apparaître comme un simple produit dérivé de ce déferlement mémoriel lié à la Réforme allemande, en rappelant tout juste sa pluralité confessionnelle.

9 Certes, l'histoire de la confession réformée n'a jamais été traitée par prétérition par la recherche allemande, d'autant qu'elle est liée à la construction du paradigme de la Konfessionnalisierung (et à la thèse de la Zweite Reformation), qui a dominé la nomenclature historique à partir des années 1980. Le paradigme, établissant un parallélisme structurel entre les trois grandes confessions chrétiennes présentes en Allemagne (catholique, luthérienne et réformée), a aussi garanti une certaine égalité de traitement entre ces dernières. Mais l'on ne peut nier que, si l'histoire de la confessionnalisation réformée a nourri de très nombreuses études en Allemagne au cours des dernières décennies, celles consacrées à la mémoire et l'histoire du Père fondateur et nomothète de la Réforme genevoise ont été moins profuses. À cela, plusieurs raisons, plus ou moins évidentes. La 
première, sans doute, est la perception du calvinisme comme phénomène allogène, introduit et surtout fortifié sur les terres d'Empire par des acteurs étrangers, identifiés pour la plupart au contingent des exilés huguenots. Dans le même temps, la Réforme luthérienne a longtemps été caractérisée comme l'expression du génie propre de la nation allemande et, même si les relents nationalistes ont bien entendu disparu de la recherche, l'impact identitaire, réel, de l'héritage luthérien n'a jamais cessé d'être souligné. La seconde raison est peut être liée à la légende noire, véhiculée par la littérature, qui entoure la figure de Calvin, dépeinte comme aussi tyrannique qu'ascétique et intolérante que croyante. Calvin, tout comme son alter ego à Wittenberg, fut très vite la cible de Vies infamantes, mais à l'inverse du "bien heureux docteur Martin Luther", peu de grands monuments littéraires et encomiastiques lui furent aussi dressés, sans doute parce que les réformés ne voulurent jamais nourrir le soupçon (en revanche bien présent concernant les luthériens) d'idolâtrer le chef historique de leur Église. Toujours est-il que les traditions diffamatoires françaises les plus diverses (du carme apostat et relaps Bolsec à la fin du XVIe siècle en passant par Richelieu, Voltaire, De Maistre ou Balzac) s'accumulèrent au fil du temps sans trouver de véritable contrepoint. En outre, elles semblèrent toutes se concrétionner dans l'œuvre de Stefan Zweig parue en 1936, Castellion contre Calvin, mettant en scène la bataille intellectuelle opposant l'apôtre vaincu de la tolérance religieuse au despote qui fit, sa vie durant, régner la terreur religieuse sur la cité lémanique ${ }^{5}$. Zweig alla même jusqu'à désigner indirectement Hitler à travers sa description de Calvin et sa condamnation de la violence fanatique.

Bien que depuis discréditée, cette aura négative de Calvin n'a jamais totalement disparu en Allemagne, et la commune préoccupation du chapelet de biographies parues à l'occasion du jubilé de sa naissance a donc été de redresser l'image déformée du Réformateur. Lors, la frontière entre la réhabilitation objective et historicisante et l'œuvre apologétique a pu être ténue, même parmi les auteurs universitaires. Des cinq principales biographies en langue allemande émanant de la recherche académique, l'une d'entre elle au moins, celle d'Hermann J. Selderhuis [11], professeur à l'Université d'Aperldoorn (Pays-Bas), a en effet été uniment critiquée par ses différents recenseurs pour sa trop apparente sympathie envers son sujet, et son usage trop généreux des citations de Calvin en guise de démonstration. Le fameux procès contre Michel Servet est par exemple analysé à la seule lumière de la correspondance de Calvin, sans laisser place aux sources (elles-mêmes abondantes) produites par l'Espagnol antitrinitaire, et surtout sans faire de place aux autres acteurs politiques de l'affaire. Tout aussi contestée est l'expression forgée par Selderhuis pour qualifier l'œuvre doctrinale de Calvin: celle d'« Ikea-Theologie ", proposée dans un sens positif par l'auteur, pour rendre compte de l'aspect combinatoire de la théologie calvinienne (puisant ses notions aussi bien chez les Pères de l'Église chrétienne, que chez Luther, Zwingli, Bucer ou Melanchthon) et de ses applications fonctionnelles simples et efficaces. Les biographies de Peter Opitz [7] (Université de Zurich), et de Wilhelm H. Neuser [6] (Université de Münster), ne disent, sur ce dernier point, pas autre chose, mais le disent autrement. Le premier insiste sur l'intense synthèse créative de Calvin, mettant en valeur l'efficacité du "nouvel espace communicatif » (Kommunikationsraum) et du "réseau relationnel » (Beziehungsnetz) de l'humanisme biblique européen. Le second, qui prend le parti de n'étudier que le «jeune Calvin ", c'est-à-dire jusqu'à l'année 1541 qui voit le retour de ce dernier à Genève, porte ainsi toute son attention à la construction progressive de la théologie calvinienne. Celle-ci se détache d'abord, selon lui, de l'influence luthérienne pour s'approcher de l'orbe mélanchthonienne, avant de dévoiler, à partir de la parution de la première Institution 
chrétienne en 1536, des accents personnels (compréhension de la Loi et de l'Évangile, prédestination) qui ne feront que s'affirmer au cours du temps. Les deux dernières Vitae calvinianae du crû 2009 s'intéressent moins à la trajectoire théologique propre de Calvin que, chez Christian Strohm [12] (Université de Heidelberg) à ses implications ecclésiales et normatives sur la pratique quotidienne de la foi ou, chez Volker Reinhard [8] (Université de Fribourg, Suisse), à ses imbrications politiques, économiques et sociales sur la « Rome réformée » du temps.

11 Notons que tous ces biographes professent au sein des facultés de théologie réformée de diverses universités, à l'exception du seul V. Reinhard, historien généraliste dont la biographie est la plus visiblement attachée au contexte politico-social de la cité lémanique qui, selon cet auteur, influença Calvin autant qu'il fut influencé par lui. Chez les historiens de l'Église (Kirchenhistoriker) en revanche, c'est essentiellement en termes de coalescence ou de disjonction doctrinale avec telle ou telle autre éminence intellectuelle de la Réforme qu'est traitée la question des influences visibles dans l'œuvre et la pensée de Jean Calvin. Or, parmi ces dernières, Melanchthon occupe une place privilégiée: l'importance de ses écrits, théologiques mais aussi philologiques et rhétoriques, vient d'être systématiquement envisagée au cours d'un colloque intitulé "Melanchthon und die reformierte Tradition", organisé à Emden à l'occasion du jubilé calvinien et... du 450ème anniversaire de la mort du Praeceptor Germaniae [17]. En 2009, une autre rencontre, proposée conjointement par les Universités de Greifswald et Tübingen avait déjà eu lieu sur le thème « Melanchthon und Calvin im Ostseeraum » [15]. Ainsi, la question du prestige de l'auteur de la Confessio variata auprès des calvinistes de la première génération, apparue il y a quelques années déjà, a été particulièrement mise à l'honneur ces derniers temps. Elle permet non seulement de réhabiliter Melanchthon comme personnalité éminente de la Réforme, et non comme simple épigone luthérien, mais aussi d'aborder de front les processus de transferts théologiques tout autant que le phénomène des transfuges confessionnels, dans la mesure où, quelques décennies après la mort de Luther, les philippistes saxons furent très vite soupçonnés d'être des cryptoréformés, des calvinistes sous le boisseau. Melanchthon semble dès lors une cheville de compréhension essentielle de la réalité plurielle du protestantisme, marquée par des effets de porosité mais aussi de contraction des frontières confessionnelles, ainsi que par la présence de zones de contact (territoriales ou mentales) amphibies particulièrement intéressantes.

Enfin, si l'on passe au crible le reste des publications parues dans le cadre du jubilé calvinien, ce sont encore des questions d'influence qui restent sur le tamis. L'influence des idées calviniennes, mais aussi du modèle ecclésial genevois, a très vite dépassé les frontières de la petite cité souveraine suisse (les confessions réformées françaises, écossaises ou le catéchisme de Heidelberg en portent bien les traces), si bien que l'on a pu parler d'Internationale calviniste essaimant à travers l'Europe et au-delà, quand dans le même temps, le luthéranisme semblait se rétracter territorialement [13, 14]. Enfin, bien sûr, c'est l'influence à long terme de la pensée de Calvin - dans ses terminaisons méthodiques - sur l'émergence de l'esprit capitaliste, mais aussi de la démocratie - dans sa germination américaine - et des libertés modernes qui, à l'occasion du jubilé, a été interrogée à nouveaux frais $[4,13]$. Or, si les analyses wébériennes sur la formation d'un ethos calviniste propre à l'émergence d'un nouvel esprit économique, tout comme le mythe (véhiculé dès le XIXe siècle par les historiens Leopold von Ranke ou George Bancroft) de Calvin «fondateur virtuel de l'Amérique » sont encore si vivement discutés, 
c'est que la recherche actuelle n'a pas renoncé à mesurer le rôle du calvinisme dans le phénomène global de la " première modernité » entamé à la fin du XVIe siècle.

Cette année 2009 dédiée à Calvin et à ses héritages aura donc produit en Allemagne des " éclats de mémoire " particulièrement brillants. Mais la stature de Calvin, tout comme les ombres qu'elle projette à travers le temps et l'espace, ménagent encore des zones plus obscures. Ainsi, entre le grand homme et les fruits lointains de son hoirie, c'est toute la culture et l'identité de la confession réformée dans les territoires allemands d'Empire, de son introduction semi-formelle à partir de la décennie 1560, en passant par sa reconnaissance juridique lors les traités de Westphalie en 1648, et au-delà, dans ses points de contact avec l'Aufklärung, et les processus de rationalisation et moralisation de la société, qui mériteraient d'être analysées plus avant. Surtout, la mémoire réformée n'est pas encore l'objet d'une histoire systématisée, comme l'est sa consœur luthérienne. Espérons donc que l'intérêt déclenché par ce jubilé se maintiendra plus longtemps qu'un feu de paille.

\section{BIBLIOGRAPHIE}

[1] Eberhard Busch, Eine Spur von Gottes Hand und Fuß. Worte von Johannes Calvin, Göttingen : Vandenhoeck \& Ruprecht, 2008

[2] Matthias Freudenberg, Georg Plasger, Calvin-Lesebuch, Neukirchen-Vluyn : Neukircher Verlag, 2008

[3] Georg Plasger, Johannes Calvins Theologie. Eine Einführung, Göttingen : Vandenhoeck \& Ruprecht, 2008

[4] Martin Ernst Hirzel, Martin Sallmann, 1509 - Johannes Calvin - 2009. Sein Werk in Kirche und Gesellschaft, Zürich : TVZ, 2008

[5] Christian Link, Johannes Calvin. Humanist, Reformator, Lehrer der Kirche, Zürich : Theologischer Verlag, 2009

[6] Wilhelm H. Neuser, Johann Calvin. Leben und Werk in seiner Frühzeit 1509-1541, Göttingen : Vandenhoeck \& Ruprecht, 2009

[7] Peter Opitz, Leben und Werk Johannes Calvins, Göttingen : Vandenhoeck \& Ruprecht, 2009

[8] Volker Reinhardt, Die Tyrannei der Tugend. Calvin und die Reformation in Genf, München : Beck, 2009

[9] Herman J. Selderhuis, Calvin Handbuch, Tübingen : Mohr Siebeck, 2008

[10] Id. (dir.), Calvinus sacrarum literarum interpres, Göttingen : Vandenhoeck \& Ruprecht, 2008

[11] Id., Johannes Calvin. Mensch zwischen Zuversicht und Zweifel. Eine Biographie, Gütersloh : Gütersloher Verlag, 2009

[12] Christoph Strohm, Johannes Calvin. Leben und Werk des Reformators, München : Beck, 2009

Expositions et colloques : 
[13] « Die Reformierten. Calvinismus in Deutschland und Europa », colloque organisée par le Deutsches Historisches Museum de Berlin (DHM), en collaboration avec la Johannes a Lasco Bibliothek d'Emden, du 16 au 18 avril 2008 à Berlin (exposition du même nom au DHM Berlin du 6 mars au 19 juillet 2009)

[14] « Calvin und Calvinismus - Europaïsche Perspektiven », colloque organisé par l'Institut für Europaïsche Geschichte de Mayence et l'Institut für Reformationsgeschichte d'Apeldoorn, du 25 au 28 juin 2009 à Mayence

[15] « Melanchthon und Calvin im Ostseeraum », colloque organisé par les Universités de Greifswald et Tübingen, du 11 au 15 novembre 2009 à Greifswald

[16] « Die andere Reformation. Johannes Calvin und die Reformierten in Mitteldeutschland», exposition du Landeshauptarchiv Sachsen Anhalt, du 6 juillet au 16 août 2010 à Dessau

[17] « Melanchthon und die reformierte Tradition », colloque organisé par la Johannes a Lasco Bibliothek d'Emden, en collaboration avec l'Université de Louvain et l'Europaïsche Melanchthonakademie de Bretten, du 10 au 12 novembre 2010

\section{NOTES}

1. L'abondance et l'ancienneté de ces références a justifié l'inclusion d'Arminius dans les « lieux de mémoire » allemands: Werner M. Doyé, «Arminius » in : Étienne François, Hagen Schulze (dir.), Deutsche Erinnerungsorte, München : Beck, 2001, vol. III, p. 587-602. C'est essentiellement sur cet article que se fonde le paragraphe qui suit

2. Signalons toutefois qu'encore en 2000, Arminius avait donné lieu à des débats animés dans les milieux politiques allemands. D'une part, la Junge Linke avait proposé le démantèlement du monument de Detmold, de manière à supprimer un ingrédient d'une fierté nationale que l'on jugeait déplacée. D'autre part, plusieurs articles polémiques s'étaient demandé - avec ironie, il est vrai - si Arminius pouvait légitimement incarner la gauche allemande. En 2009, toutefois, ces arguments ne furent plus employés

3. Les références des trois volumes, sous le titre commun 2.000 Jahre Varuschlacht, sont les suivantes: Rudolf Aßkamp et Herwig Kenzler (dir.), Imperium, Darmstadt: Wissenschaftliche Buchgesellschaft, 2009 ; Rudolf Aßkamp et Stefan Burmeister (dir.), Konflikt, eod. loc., 2009 ; Rudolf Aßkamp et Herwig Kenzler (dir.), Mythos, eod. loc., 2009. Le site Internet de l'exposition peut être consulté ici : http://www.imperium-konflikt-mythos.de (consulté le 16 août 2010).

4. Dieter Timpe, Arminius-Studien, Heidelberg : Winter, 1970

5. On lira, sur ce point, l'article de Frank Lestringant, «Stefan Zweig contre Calvin (1936)» in : Revue de l'histoire des religions, 2006/1, p. 71-94.

\section{AUTEURS}

\section{MARION DESCHAMP}

Marion Deschamp est boursière d'aide à la mobilité à l'IFHA. 


\section{THOMAS LIENHARD}

Thomas Lienhard est directeur de l'IFHA. 